\title{
Pseudomonas aeruginosa transmission is infrequent in New Zealand cystic fibrosis clinics
}

\author{
J. Schmid*, L.J. Ling*, J.L.S. Leung*, N. Zhang*, J. Kolbe ${ }^{\#}$, A.W. Wesley \\ G.D. Mills ${ }^{+}$, P.J. Brown ${ }^{\S}$, D.T. Jones ${ }^{f}$, R.T.R. Laing**, P.K. Pattemore ${ }^{\# \#,}$ \\ D.R. Taylor ${ }^{\text {Tी }}$ and K. Grimwood ${ }^{++}$
}

ABSTRACT: Pseudomonas aeruginosa is an important pathogen in cystic fibrosis (CF). Although most patients harbour unique $P$. aeruginosa isolates, some clinics report patients sharing common strains. The overall importance of person-to-person transmission in $P$. aeruginosa acquisition and whether routine patient segregation is necessary remains uncertain. The present authors therefore investigated the extent of $P$. aeruginosa transmission in New Zealand CF clinics.

New Zealand's seven major CF centres were assessed, combining epidemiological data with computer-assisted Sall DNA fingerprinting of $\mathbf{4 9 6}$ isolates from 102 patients.

One cluster of related isolates was significantly more prevalent in the largest clinic than expected by chance. The seven patients with isolates belonging to this cluster had more contact with each other than the remaining patients attending this centre. No other convincing evidence of transmission was found in any of the other smaller clinics. Three $P$. aeruginosa strains believed to be transmissible between patients in Australian and British CF clinics are present in New Zealand, but there was no definite evidence they had spread.

Pseudomonas aeruginosa transmission is currently infrequent in New Zealand cystic fibrosis clinics. This situation could change rapidly and ongoing surveillance is required. The current results confirm that computer-assisted Sall DNA fingerprinting is ideally suited for such surveillance.

KEYWORDS: Cystic fibrosis, DNA typing, Pseudomonas aeruginosa, transmission

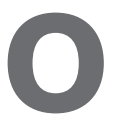

nce Pseudomonas aeruginosa is established within the lungs of patients with cystic fibrosis (CF) there is an accelerated decline in pulmonary function, quality of life and life-expectancy [1]. Most CF patients acquire $P$. aeruginosa strains from environmental sources [2]. However, siblings are often infected with indistinguishable clones suggesting person-toperson transmission instead of common environmental exposure [3]. Furthermore, in several European, English and Australian CF clinics, many patients share a common strain $[2,4-7]$. Some strains appear in several clinics $[8,9]$, others superinfect patients already chronically colonised with unrelated $P$. aeruginosa isolates [10], while some are associated with worse outcomes and increased treatment costs $[6,7$, 11-13]. In contrast, others have found only small clusters of genetically similar isolates from
CF patients lacking geographic or temporal relationships other than attending the same clinic [14-16]. Rather than from cross-infection, such clusters could be due to common environmental sources or limited discriminatory power of typing methods [17].

Consequently, $P$. aeruginosa transmission between CF patients may occur, but only after prolonged close contact, as in siblings, or from small numbers of strains with heightened potential for transmission. Nonetheless, British and, more recently, Australian CF Trust infection control guidelines and the European consensus report recommend that all CF patients with $P$. aeruginosa be segregated to reduce crossinfection [18-20]. Centres in Australia and Europe that implemented these recommendations have been successful at restricting potentially transmissible strains [21, 22]. However,
AFFILIATIONS

*Institute of Molecular Biosciences, Massey University, and ${ }^{\text {s}}$ Dept of Paediatrics, Palmerston North Hospital, Palmerston North "Dept of Medicine, University of Auckland, and

'Paediatric Gastroenterology Service Starship Children's Health, Auckland, +Dept of Infectious Disease, Waikato Hospital, Hamilton,

${ }^{f}$ Respiratory Medicine, Wellington Hospital, and

${ }^{++}$Dept of Paediatrics and Child

Health, University of Otago,

Wellington,

**Respiratory Services, Christchurch Hospital, and

\#\#Dept of Paediatrics, University of Otago, Christchurch, and

"Otago Respiratory Research Unit, University of Otago, Dunedin, New Zealand

CORRESPONDENCE

K. Grimwood, Director of Research, Royal Children's Hospital, Herston Road, Herston, Queensland 4029, Australia. Fax: 61736365578 E-mail: Keith_Grimwood@ health.qld.gov.au

Received:

July 012008

Accepted after revision:

August 012008

SUPPORT STATEMENT

The New Zealand Child Health

Research Foundation (Auckland, New Zealand) provided financial support for the present study.

STATEMENT OF INTEREST None declared. 
segregation-based infection control policies are expensive and difficult to implement, and adversely affect patient well-being without reducing sporadic $P$. aeruginosa acquisition [22]. It is therefore important to determine whether cross-infection is occurring within individual clinics.

Previously, a computer-assisted DNA fingerprinting method was described, which is simple, fast and cost-effective [23]. The DNA fingerprints are stable [23], the pattern score-based genetic distances correlate with DNA sequence-based genetic distances [23], and its discriminatory power matches that of pulse-field gel electrophoresis (PFGE), the current gold standard for molecular typing $P$. aeruginosa isolates [23-25]. A robust pattern scoring system used with the DNA fingerprinting technique largely overcomes problems associated with noncategorical molecular typing methods [17]. Previously, using computer-assisted SalI fingerprinting, indications of possible transmission in one New Zealand clinic were found based upon $P$. aeruginosa isolates from CF patients being genetically less diverse than isolates obtained from patients without CF [23]. However, it was not directly established that this resulted from CF clinic attendance. Therefore, a multi-centre study was conducted to determine the likelihood of P. aeruginosa transmission in New Zealand CF clinics by combining epidemiological data with SalI fingerprinting. The clinics were located in cities 100-1,100 km apart. Each employed standard hygiene methods, but most had not implemented patient segregation.

\section{METHODS}

\section{Patients, data collection and isolates}

Participants attended one out of seven (table 1) clinics caring for 269 CF patients (102 adults aged $\geqslant 15$ yrs; clinic size $21-57$ ), representing $71 \%$ of child, $84 \%$ of adult and $75 \%$ of total New
Zealand CF patients. Treatment and infection control protocols were similar between hospitals [26]. Inpatients were nursed either in single rooms with their own bathrooms or in wards with non-CF patients. Contact between CF patients was discouraged and respiratory equipment was not shared. In-line filters, mouthpieces and connectors for pulmonary function equipment were discarded or sterilised after use, and physiotherapy was conducted separately. Segregation of all CF individuals in outpatient clinics was practised by Starship Children's Health (Auckland), Christchurch Hospital (paediatric clinic only; Christchurch) and Dunedin hospitals (Dunedin, New Zealand). The other hospitals segregated only those harbouring Burkholderia cepacia complex or methicillin-resistant Staphylococcus aureus. The Ministry of Health's Multi-Region Ethics Committee (Wellington, New Zealand) approved the study as a clinical audit.

Following informed consent, single sputum or oropharyngeal specimens were collected from CF patients attending routine clinic appointments between July 2004 and August 2005. The patient's physician entered centre, age, sex, sputum microbiology and, for the previous 12 months, dates of hospitalisation and clinic attendances, best recorded body mass index (BMI) z-scores and forced expiratory volume in one second (FEV1) \% predicted values into a secure database.

Respiratory samples were inoculated on selective media to detect CF respiratory pathogens by standard techniques [27]. When $P$. aeruginosa was identified, five randomly selected colonies were each inoculated into glycerol broth and stored at $-70^{\circ} \mathrm{C}$ (see online supplementary material).

Representative isolates of clonal strains from CF patients in Liverpool [4] and Manchester [5] (both UK) and Melbourne

TABLE 1 Patient population and isolates

\begin{tabular}{|c|c|c|c|c|c|c|c|c|c|}
\hline \multirow[t]{2}{*}{ Clinic $^{\#}$} & \multirow{2}{*}{$\begin{array}{c}\text { Patients } \\
\text { sampled } n\end{array}$} & \multirow{2}{*}{$\begin{array}{c}\text { Isolates } \\
\text { fingerprinted } \\
n\end{array}$} & \multirow{2}{*}{$\begin{array}{c}\text { Timeframe of } \\
\text { collection } \mathrm{mm} / \\
\text { yyyy }^{+}\end{array}$} & \multicolumn{6}{|c|}{ Patient pairs in contact category $\$$} \\
\hline & & & & I & II & III & IV & v & VI \\
\hline Starship & 8 & 39 & $11 / 2004-05 / 2005$ & $1(4)$ & $4(14)$ & $15(54)$ & $5(18)$ & $0(0)$ & $3(11)$ \\
\hline Greenlane & 38 & 182 & $11 / 2004-03 / 2005$ & $1(0)$ & $25(4)$ & $136(19)$ & $59(8)$ & $95(14)$ & $387(55)$ \\
\hline Waikato & 5 & 24 & 02/2005-06/2005 & $0(0)$ & $0(0)$ & $0(0)$ & $0(0)$ & $3(30)$ & $7(70)$ \\
\hline Christchurch & 17 & 81 & 11/2004-05/2005 & $0(0)$ & $10(7)$ & $6(4)$ & $12(9)$ & $16(12)$ & $92(68)$ \\
\hline Dunedin & 10 & 44 & 12/2004-08/2005 & $1(2)$ & $0(0)$ & $7(16)$ & $6(13)$ & $3(7)$ & $28(62)$ \\
\hline
\end{tabular}

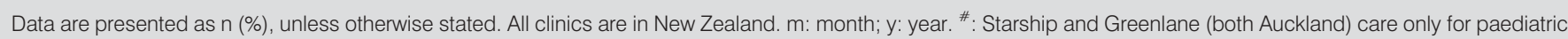
and adult cystic fibrosis (CF) patients, respectively. The other centres care for both adult and paediatric patients, but in separate facilities at each centre. All clinics except Starship and Greenlane are located $>100 \mathrm{~km}$ from each other. ": two patients moved during the study and were sampled in two different centres. These are counted as sampled patients in both centres. ${ }^{+}:$for each centre, most $(81 \%)$ respiratory specimens were collected within a 4-month period. ${ }^{5}$ : for each patient the amount of contact with every other patient in the study in the 12 months preceding sample collection was assessed by CF clinic staff and based upon sibship, clinic attendance and hospitalisation. A matrix was generated which described the amount of contact between all possible pairs of patients in a given centre using arbitrarily formed contact categories, also referred to as physical distance categories. I: siblings; II: patient pairs hospitalised in the same ward at overlapping times; III: patient pairs who attended outpatient clinics on the same day; IV: patient pairs where one attended an outpatient clinic at the same time the other was hospitalised; V: patient pairs who were hospitalised, or attended outpatient clinics, but never at the same time; VI: patient pairs who were not hospitalised and never attended clinics at the same time. All contact categories were defined on the basis of outpatient clinic attendance and hospitalisation data in the 12 months preceding Pseudomonas aeruginosa isolate collection. Percentage values are given relative to all patient pairs in categories I-VI. 
(two pulsotype1 isolates) [6] and Brisbane (pulsotype2; both Australia) [7] were kindly provided by J. Govan, University of Edinburgh (Edinburgh, UK), A. Jones, Manchester Adult CF Centre (Manchester, UK), D. Armstrong, Monash University (Melbourne, Australia) and S. Bell, the Prince Charles Hospital (Brisbane, Australia), respectively.

\section{P. aeruginosa genotyping}

DNA fingerprinting was performed as described previously [23]. Briefly, following electrophoresis and ethidium bromide staining of SalI digests of genomic DNA, the number of restriction fragments in molecular weight brackets (defined by an XV molecular weight standard (Roche Diagnostics, Auckland, New Zealand) loaded in alternate lanes) was counted for each strain, and used to calculate genetic distances (see online supplementary material). Distances were displayed as unweighted pair-group method with arithmetic mean (UPGMA) trees using Paup*4.0 (Sinauer Associates, Sunderland, MA, Australia). Five colonies per patient were typed. PFGE of SpeI-genomic DNA digests of P. aeruginosa isolates was also performed [28].

\section{Statistical analysis}

Simple descriptive statistics, binomial, unpaired t- and Fisher's exact tests were performed. Statistical significance was determined by $\mathrm{p}<0.05$ and Bonferroni correction applied when multiple comparisons were made.

\section{RESULTS}

\section{Frequency of P. aeruginosa in the study population}

Out of the 269 patients attending seven clinics, 102 (38\%) had $P$. aeruginosa in sputum $(n=97)$ or oropharyngeal $(n=5)$ cultures. The characteristics of these 102 patients are shown in table 2 .

\section{Evidence for transmission at one centre}

Table 1 shows that $496 \mathrm{P}$. aeruginosa isolates from $102 \mathrm{CF}$ patients underwent DNA fingerprinting. This included two patients who moved during the study and provided samples in two clinics. Table 1 also displays the degree of contact between patients. Figure 1 shows the relationships between isolates as a UPGMA tree. If a patient had several isolates with identical patterns, only one of these is included in the tree. Four strains highly prevalent in CF clinics overseas are also included.

Transmission results in clusters of identical or very similar DNA types in different patients. To arrive at a meaningful threshold for identifying clusters, the genetic distance between two independent scores of the same pattern was determined in 50 instances, in two different DNA preparations. The average genetic distance was 0.094 ; the upper $95 \%$ confidence limit was 0.118 . Therefore, 0.118 was used to define clusters and above this distance the present authors were confident of distinguishing different patterns.

In 67 patients, all isolates were identical or could be assigned to one cluster specific to this patient (grey boxes in fig. 1) indicating that their isolates were derived from a single infecting strain. Isolates from the remaining 35 patients were too different to be placed into single patient-specific clusters (although often they were still more similar to each other than to isolates from any other patient). For the two patients who moved during the study and were sampled twice, GRE142/ DUN242 and GRE144/WAI249 (lower case isolate labels in fig. 1), isolates obtained at both centres fell into the same clusters, with one exception (gre144 3).

Some clusters contained isolates from two to nine different patients (black boxes in fig. 1). Such clustering can result from transmission, clonal expansions predating and unrelated to transmission in the patient population under study, or simply because no typing method produces an infinite number of types [17]. To estimate how much clustering is expected without transmission in the current patient population, the frequency of distance values $<0.118$ was calculated when only isolates obtained at different hospitals were compared (i.e. isolates lacking direct epidemiological connection; $1.9 \%$; 336 out of 17,078 comparisons). Therefore without transmission, $1.9 \%$ of distance values resulting from all comparisons between isolates from different patients in figure 1 should be $<0.118$. However, $2.2 \%$ ( 486 out of 21,366 ) of comparisons from patients in figure 1 had distance values $<0.118$; 65 more than expected without transmission.

The present study next assessed if any of the clusters in figure 1 involved a significantly higher proportion of patients from one centre, as expected if a cluster were resulting from transmission. This was the case only for the largest cluster (involving nine patients), isolated almost exclusively from patients attending the Greenlane CF Centre (seven out of 38 Greenlane patients had isolates that were part of this cluster,

\begin{tabular}{|c|c|c|}
\hline TABLE 2 & \multicolumn{2}{|c|}{$\begin{array}{l}\text { Characteristics of patients with Pseudomonas } \\
\text { aeruginosa }\end{array}$} \\
\hline \multicolumn{3}{|c|}{ Proportions of patients with $P$. aeruginosa } \\
\hline Adults $\geqslant 15$ & & $83 / 102(81)$ \\
\hline Children $<$ & yrs & 19/167 (11) \\
\hline Total & & 102/269 (38) \\
\hline \multicolumn{3}{|c|}{ Patients with $P$. aeruginosa ${ }^{\#}$} \\
\hline Age yrs & & $20.5(17-29)$ \\
\hline Age range & & $6-65$ \\
\hline Males & & 67 \\
\hline Sibling pair & & 5 \\
\hline Patients wit & first acquisition & 4 \\
\hline Duration of & fection yrs & $0-\geqslant 10$ \\
\hline \multicolumn{3}{|c|}{ Colonial morphology } \\
\hline Mucoid & & 27 \\
\hline Nonmuco & & 26 \\
\hline Mucoid a & d nonmucoid & 38 \\
\hline Co-pathoge & & 75 \\
\hline Staphyloc & ccus aureus ${ }^{\S}$ & 64 \\
\hline Haemoph & us influenzae & 13 \\
\hline Stenotrop & omonas maltophilia & 9 \\
\hline Burkholde & a cepacia complex & 3 \\
\hline Others ${ }^{f}$ & & 30 \\
\hline \multicolumn{3}{|c|}{$\begin{array}{l}\text { Data are presented as } n / \text { total (\%), median (interquartile range), range or } n \text {. } \\
\text { \#: } n=102 ;{ }^{\bullet}: n=91 ;+: 75 \text { patients were infected with } 1-3 \text { respiratory pathogens; } \\
\text { s: all isolates were methicillin-susceptible; }{ }^{f:}: 21 \text { Aspergillus species, five other } \\
\text { Gram-negative bacilli and four nontuberculous mycobacteria. }\end{array}$} \\
\hline
\end{tabular}



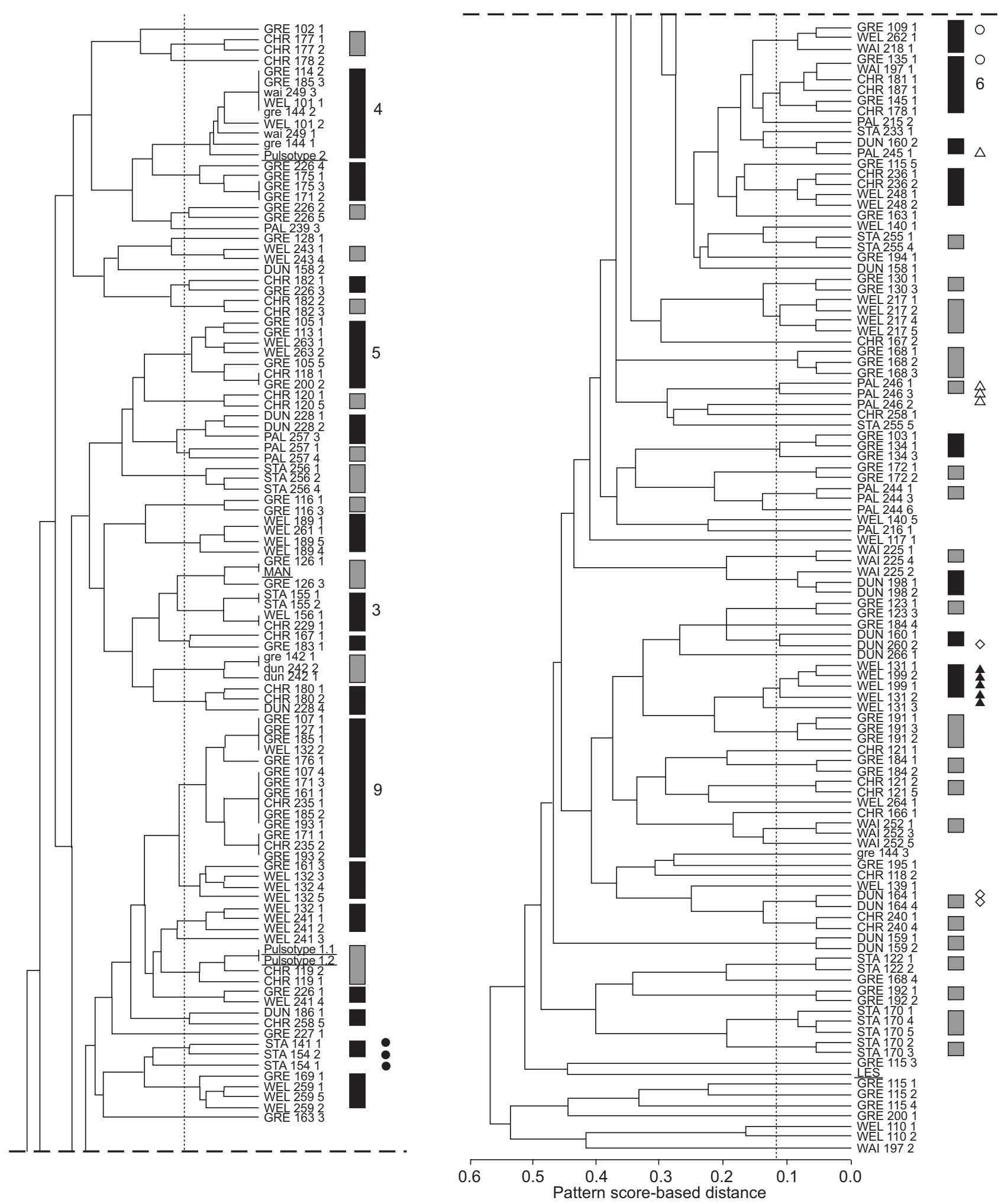

FIGURE 1. Unweighted pair-group method with arithmetic mean tree based on Sall pattern score-based genetic distances between representative isolates (where multiple isolates from the same patients are scored as identical, only one representative is included). Isolate labels reflect the clinic in New Zealand at which a patient was treated (Greenlane (GRE; Auckland); Starship (STA; Auckland); Waikato (WAl; Hamilton); Palmerston North (PAL); Wellington (WEL); Christchurch (CHR); and Dunedin (DUN)) followed by a three-digit patient number. The fourth digit indicates which of the five isolates from the patient is shown. Lower-case isolate names highlight two cases where patients were sampled twice, at different centres. Isolates belonging to overseas strains are from: Liverpool, UK (LES); Manchester, UK (MAN); Melbourne, Australia (pulsotype1.1 and pulsotype1.2) and Brisbane, Australia (pulsotype2). $\bullet, \bigcirc, \mathbf{\Delta}, \triangle, \diamond$ : isolates obtained from five sibling pairs. ……: threshold genetic distance of 0.118 used for defining clusters of isolates with highly similar patterns. Grey bars show clusters present in only one New Zealand patient. Black bars show clusters present in several New Zealand patients. In more than two where a cluster was present in more than two New Zealand patients, the number of patients is shown to the right of the bar 
compared with two out of 66 patients from other clinics; Fisher's exact test $\mathrm{p}=0.012$ ). Also, patients with isolates belonging to the cluster had more centre-related contact with each other than the remaining Greenlane patients (fig. 2; $p=0.015$; the sum of 21 pairwise physical distance category values for the seven patients was lower than the sum of 986 out of 1,000 sets of 21 values chosen randomly from a matrix of all remaining Greenlane patient pairwise physical distance category values), consistent with the hypothesis that transmission was healthcare-related.

No significant (unpaired t-test) differences were found in several health status indicators (BMI, z-scores, FEV1 values, frequency of clinic attendance or days in hospital) between the nine patients carrying strains belonging to this shared cluster and all other study patients, or between the seven Greenlane patients carrying these strains and all other Greenlane patients. Also, the sex or age distribution of patients infected with cluster isolates did not differ significantly (Fisher's exact and unpaired t-tests) from that of the remaining patients (data not shown).

\section{Prevalence of potentially transmissible overseas strains in New Zealand}

The introduction and spread of highly transmissible overseas strains is a potential threat to New Zealand patients. Therefore, representatives of four such strains were included in the current analysis to examine whether such strains might be spreading into New Zealand. New Zealand isolates clustering with three of the strains were found. Isolates with SalI types highly similar to the overseas strains also had PFGE types indistinguishable or closely related to those of the overseas strains (see online supplementary material; fig. 1). The Manchester and pulsotype1 strains each clustered with isolates from a single New Zealand patient. The adult (GRE126) with

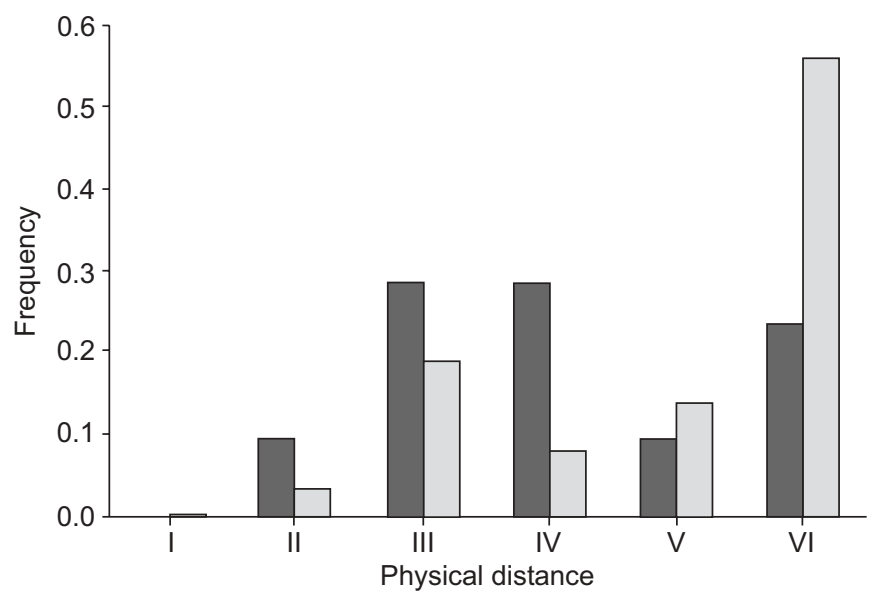

FIGURE 2. Distribution of physical distance categories among Greenlane patients contributing to the largest cluster ( $\square$ ) and the remaining Greenlane patients ( $\square$ ). I: siblings; II: patient pairs hospitalised in the same ward at overlapping times; III: patient pairs who attended outpatient clinics on the same day; IV: patient pairs where one attended an outpatient clinic at the same time the other was hospitalised; V: patient pairs who were hospitalised, or attended outpatient clinics, but never at the same time; VI: patient pairs who were not hospitalised and never attended clinics at the same time. an isolate indistinguishable from the Manchester strain had lived in London, UK, and it was there his initial P. aeruginosa infection was first detected. Another patient (CHR119) possessed isolates similar to Melbourne pulsotype1 isolates and had previously holidayed in Queensland, Australia, although without making contact with Australian CF clinics or patients. Brisbane pulsotype2 isolates formed a cluster with isolates from four New Zealand patients. Two patients (WEL101 and GRE144/WAI249) had previously lived in Brisbane where one first acquired $P$. aeruginosa before immigrating to New Zealand. Both had mixed freely with Australian CF patients while receiving treatment. The remaining two patients (GRE114, GRE185) had isolates resembling pulsotype 2 and were treated in the same centre as, but were never in direct contact with, a patient previously exposed to pulsotype2 overseas (GRE144/WAI249). Thus at most three patients are likely to have acquired overseas strains in New Zealand.

\section{Centre-related patient contact and distance between their isolates}

Many of the centres in the present study had very small patient numbers, and in these transmission may only lead to very small clusters, and thus be difficult to detect by assessing clustering. Therefore, an alternative approach of searching for transmission was also used, by determining whether the genetic distance between patients' isolates tends to decrease with increased patient contact.

It was first determined if the closest-related counterparts of isolates from patients in a given clinic were isolates from other patients at the same clinic more often than expected by chance. This was not the case (see online supplementary material; table 1). Next it was checked whether, within each clinic, the average genetic distance between patients' isolates decreased as the degree of physical contact (and opportunity of transmission) increased (fig. 3). For comparison in the analysis the distribution of genetic distances between isolates from the same patient and the distribution of genetic distances between isolates from a given centre and isolates from all other centres were included. Only isolates from different siblings were generally more similar to each other than isolates from pairs of patients treated in different centres, indicating that the multiple $P$. aeruginosa isolates in most sibling pairs were derivatives of a single strain. In contrast, the distribution of genetic distances between isolates from pairs of patients with various degrees of contact through treatment at the same centre was not significantly different from that between isolates from patient pairs treated at different clinics, although at Greenlane the median genetic distances, in all but one of these categories, were lower than those between isolates at Greenlane and those from patients at all other hospitals. The current study also failed to detect a dependence on the frequency of low genetic distance values on centre-related patient contact (see online supplementary material).

Lastly, it was determined whether the four patients who first acquired $P$. aeruginosa during the course of the present study (WEL110, CHR235, WELL243 and PAL257) harboured isolates closely related to those from other CF patients at the same clinic. In each case the isolates in the current sample closest related to theirs were not from the same centre. 

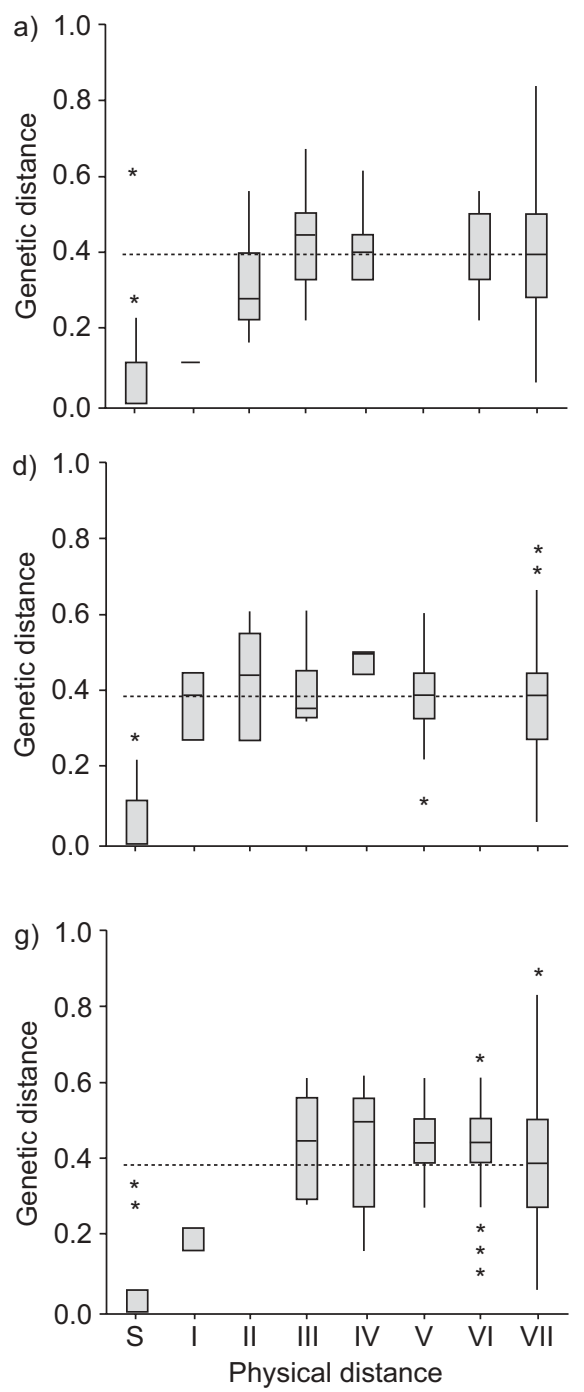

b)

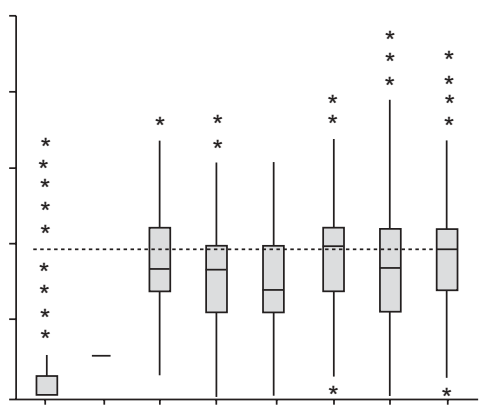

e)

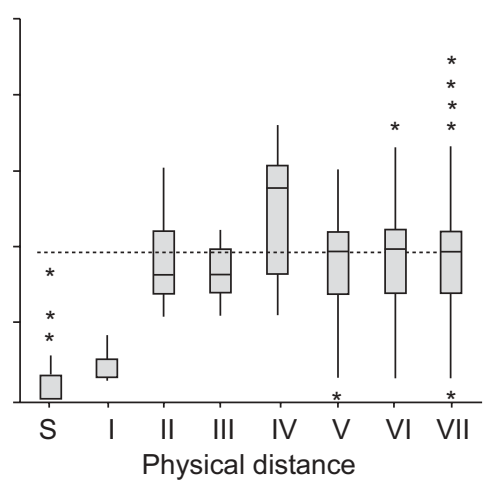

c)

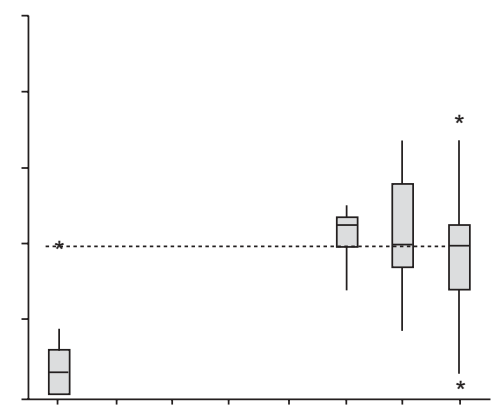

f)

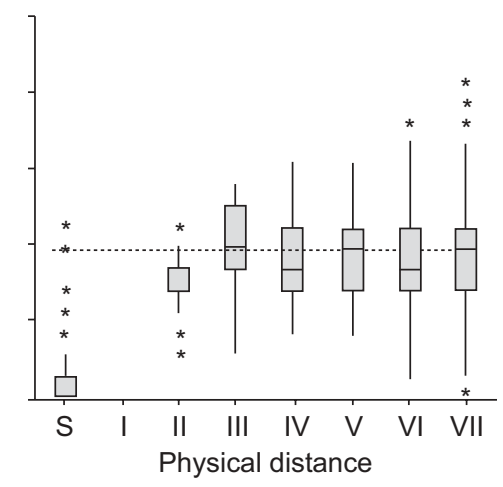

FIGURE 3. Box and whisker plots of the distribution of genetic distances between isolate pairs from patients with different degrees of physical contact in New Zealand cystic fibrosis clinics: a) Starship (Auckland); b) Greenlane (Auckland); c) Waikato (Hamilton); d) Palmerston North; e) Wellington; f) Christchurch; and g) Dunedin. S: pairs of isolates from the same patient; I: siblings; II: patient pairs hospitalised in the same ward at overlapping times; III: patient pairs who attended outpatient clinics on the same day; IV: patient pairs where one attended an outpatient clinic at the same time the other was hospitalised; V: patient pairs who were hospitalised, or attended outpatient clinics, but never at the same time; VI: patient pairs who were not hospitalised and never attended clinics at the same time; VII: pairs of isolates from pairs of patients treated in different hospitals, i.e. the genetic distances in category VII are those between isolates obtained in the hospital the figure refers to and isolates in all other hospitals. ......... median of genetic distances in category VII.

\section{DISCUSSION}

Transmission of $P$. aeruginosa between New Zealand CF patients appears infrequent. Isolates from patient pairs with frequent treatment-related contact are generally not more closely related than those from patients attending different centres. This also indicates that, with the possible exception of siblings who often share similar strains, social contact generally did not lead to significant transmission: otherwise, isolates from patients treated in the same centre should be more closely related than isolates from patients treated in different centres hundreds of kilometres apart.

The only evidence for healthcare-related transmission is a cluster of isolates in seven out of 38 patients at Greenlane. The cluster is overrepresented in Greenlane where patients with isolates belonging to the cluster were in closer contact with one another than other Greenlane patients. Also the cluster's size (97 distance values $<0.118$ among cluster isolates from Greenlane) roughly matches the excess of distance values $<0.118$ in the present patient population indicative of transmission.
Evidence for transmission is also consistent with the current authors' earlier findings [23] of reduced genetic diversity of isolates from CF patients compared with isolates from other Greenlane patients, but together the two studies also indicate that transmission is uncommon at Greenlane. The cluster's prevalence has not changed significantly in the 8 yrs since the earlier study, when cluster isolates were present in three out of 20 CF patients [23], and had not increased among five patients participating in both studies where one of these patients (GRE 107) had cluster isolates on both occasions. Furthermore, three out of the remaining four patients (GRE102, 105, 123 and 145) also retained their original strains. Likewise the pulsotype2 strain, capable of spreading overseas, had not done so in Greenlane to a readily demonstrable degree. Two patients may have acquired the strain while at Greenlane. However, no direct epidemiological connection was identified, and the main Greenlane cluster can account for all transmission-related clustering in the present sample, arguing against transmission as a cause of the pulsotype 2 cluster. Thus, because typing methods do not give an unlimited number of patterns and sample only a small part of the genome [17, 23], the pulsotype2 
isolates from the two patients may only be similar to, but not derived from, the overseas pulsotype 2 strain.

Current infection control measures thus appear to protect patients from transmission in New Zealand clinics, except for the cohort presently attending Greenlane where a recent report also suggested cross-infection by Burkholderia multivorans may have taken place [29]. When interpreting these data, the small size of New Zealand CF clinics must be considered. Patients in smaller centres usually have less contact with one another [30] and thus less opportunity to transmit strains. Certainly, the clinic where the evidence was found for transmission was by far the largest in the current study, with substantially more patient contact compared with other centres. The present results raise the possibility that the existing body of research, based largely on findings in large CF clinics, may overestimate the risk of cross-infection among the many CF patients who are still treated in smaller centres. Furthermore, smaller centres also make it harder to detect transmission by visual inspection of dendrograms. However, the analysis in figure 3 and the current analysis based on the expected frequency of distance values $<0.118$ in the entire sample without epidemiological contact should have revealed any transmission occurring in other centres. It could be argued that the latter analysis may have underestimated transmission among the present study patients, since its calculations are based on the assumption that patients treated in different centres lack any epidemiological connection. This is not entirely accurate, as patients do move between clinics. Two such movements were recorded during the present study and additional movement will have occurred before the investigation. However, the error in the current estimated frequency of distance values $<0.118$ between unconnected isolates is probably small. It had been determined earlier [23] that the discriminatory power of the current method (probability that two unrelated isolates can be discriminated), was 0.99 , using isolates from unrelated nonCF patients where unexpected epidemiological relationships are very unlikely. In the current study, $1.9 \%$ of pair-wise comparisons between representative isolates from patients treated in different centres were not reliably distinguishable from one another (genetic distance $<0.118$ ), equivalent to a discriminatory power of 0.98 . This means that isolates from different centres in the present study were approximately as epidemiologically unconnected as isolates from epidemiologically unrelated non-CF patients.

This new estimate of discriminatory power, the finding that SalI patterns are stable over 7-8 yrs and observing isolates that formed clusters with the present method were also indistinguishable by pulse-field gel electrophoresis, all confirm SalI typing as an attractive alternative to pulse-field gel electrophoresis, in particular if cost is considered as well. The current study indicates that surveillance of the total New Zealand cystic fibrosis population would cost $\sim$ US\$8,000 per year.

\section{ACKNOWLEDGEMENTS}

The present authors would like to thank A. Farrell, D. Graham, P. Jackson, M. O'Carroll, M. Tweed, A. Watson, M. Wilsher, M. Hoggard, V. Isles, J. Tate, L. Thrift, K. Brassett and A. Williamson for assistance with recruitment, sample and data collection; M. Bilkey, C. Brehmer, J. DeLores, H. Maguire,
L. McKnight, J. Parker, C. Pickett, R. Podmore, K. Stockman and A. Van Der Linden for identification of isolates; U. von Mulert for database design; A. Grinberg and R. Pattison for assistance with PFGE; A. Morris for comments on the manuscript; and A. Palamountain for programming.

\section{REFERENCES}

1 Emerson J, Rosenfeld M, McNamara S, Ramsey B, Gibson RL. Pseudomonas aeruginosa and other predictors of mortality and morbidity in young children with cystic fibrosis. Pediatr Pulmonol 2002; 34: 91-100.

2 Romling U, Kader A, Sriramulu DD, Simm R, Kronvall G. Worldwide distribution of Pseudomonas aeruginosa clone $\mathrm{C}$ strains in the aquatic environment and cystic fibrosis patients. Environ Microbiol 2005; 7: 1029-1038.

3 Grothues D, Koopmann U, von der Hardt H, Tummler B. Genome fingerprinting of Pseudomonas aeruginosa indicates colonization of cystic fibrosis siblings with closely related strains. J Clin Microbiol 1988; 26: 1973-1977.

4 Cheng K, Smyth RL, Govan JR, et al. Spread of beta-lactamresistant Pseudomonas aeruginosa in a cystic fibrosis clinic. Lancet 1996; 348: 639-642.

5 Jones AM, Govan JR, Doherty CJ, et al. Spread of a multiresistant strain of Pseudomonas aeruginosa in an adult cystic fibrosis clinic. Lancet 2001; 358: 557-558.

6 Armstrong DS, Nixon GM, Carzino R, et al. Detection of a widespread clone of Pseudomonas aeruginosa in a pediatric cystic fibrosis clinic. Am J Respir Crit Care Med 2002; 166: 983-987.

7 O'Carroll MR, Syrmis MW, Wainwright CE, et al. Clonal strains of Pseudomonas aeruginosa in paediatric and adult cystic fibrosis units. Eur Respir J 2004; 24: 101-106.

8 Armstrong D, Bell S, Robinson M, et al. Evidence for spread of a clonal strain of Pseudomonas aeruginosa among cystic fibrosis clinics. J Clin Microbiol 2003; 41: 2266-2267.

9 Scott FW, Pitt TL. Identification and characterization of transmissible Pseudomonas aeruginosa strains in cystic fibrosis patients in England and Wales. J Med Microbiol 2004; 53: 609-615.

10 McCallum SJ, Corkill J, Gallagher M, Ledson MJ, Hart CA, Walshaw MJ. Superinfection with a transmissible strain of Pseudomonas aeruginosa in adults with cystic fibrosis chronically colonised by $P$ aeruginosa. Lancet 2001; 358: 558-560.

11 Nixon GM, Armstrong DS, Carzino R, et al. Clinical outcome after early Pseudomonas aeruginosa infection in cystic fibrosis. J Pediatr 2001; 138: 699-704.

12 Al-Aloul M, Crawley J, Winstanley C, Hart CA, Ledson MJ, Walshaw MJ. Increased morbidity associated with chronic infection by an epidemic Pseudomonas aeruginosa strain in CF patients. Thorax 2004; 59: 334-336.

13 Jones A, Dodd M, Doherty C, Govan J, Webb A. Increased treatment requirements of patients with cystic fibrosis who harbour a highly transmissible strain of Pseudomonas aeruginosa. Thorax 2002; 57: 924-925.

14 Speert DP, Campbell ME, Henry DA, et al. Epidemiology of Pseudomonas aeruginosa in cystic fibrosis in British Columbia, Canada. Am J Respir Crit Care Med 2002; 166: 988-993. 
15 Van daele S, Vaneechoutte M, De Boeck K, et al. Survey of Pseudomonas aeruginosa genotypes in colonised cystic fibrosis patients. Eur Respir J 2006; 28: 740-747.

16 Da Silva Filho LV, Levi JE, Bento CN, Rodrigues JC, Da Silva Ramos SR. Molecular epidemiology of Pseudomonas aeruginosa infections in a cystic fibrosis outpatient clinic. J Med Microbiol 2001; 50: 261-267.

17 Blanc DS. The use of molecular typing for epidemiological surveillance and investigation of endemic nosocomial infections. Infect Genet Evol 2004; 4: 193-197.

18 Cystic Fibrosis Australia and Hooker LJ. Cystic Fibrosis Australia Infection Control Guidelines for Cystic Fibrosis Patients and Carers. Sydney, Cystic Fibrosis Australia, 2007. www.cysticfibrosis.org.au/pdf/Handbook-Infection_ Control.pdf Date last updated: April 2007.

19 CF Trust Infection Control Group. Pseudomonas aeruginosa Infection in People with Cystic Fibrosis: Suggestions for Prevention and Infection Control. 2nd Edn. Bromley, Cystic Fibrosis Trust, 2004. www.cftrust.org.uk/aboutcf/ publications/consensusdoc/C_Pseudomonas_aeruginosa_ Nov_04.pdf Date last updated: November 2004.

20 Doring G, Hoiby N, Consensus Study Group. Early intervention and prevention of lung disease in cystic fibrosis: a European consensus. J Cyst Fibros 2004; 3: 67-91.

21 Jones AM, Dodd ME, Govan JR, et al. Prospective surveillance for Pseudomonas aeruginosa cross-infection at a cystic fibrosis center. Am J Respir Crit Care Med 2005; 171: 257-260.

22 Griffiths AL, Jamsen K, Carlin JB, et al. Effects of segregation on an epidemic Pseudomonas aeruginosa strain in a cystic fibrosis clinic. Am J Respir Crit Care Med 2005; 171: 1020-1025.
23 Al-Samarrai TH, Zhang N, Lamont IL, et al. Simple and inexpensive but highly discriminating method for computer-assisted DNA fingerprinting of Pseudomonas aeruginosa. J Clin Microbiol 2000; 38: 4445-4452.

24 Silbert S, Pfaller MA, Hollis RJ, Barth AL, Sader HS. Evaluation of three molecular typing techniques for nonfermentative Gram-negative bacilli. Infect Control Hosp Epidemiol 2004; 25: 847-851.

25 Grundmann H, Schneider C, Hartung D, Daschner FD, Pitt TL. Discriminatory power of three DNA-based typing techniques for Pseudomonas aeruginosa. J Clin Microbiol 1995; 33: 528-534.

26 Wesley AW, Asher MI, Gillies JD, Pattemore PK, Kerr A, Hewitt C. Proposals for standards of cystic fibrosis management in New Zealand. A position statement by the Respiratory Committee of the Paediatric Society of New Zealand. N Z Med J 1996; 109: 140-141.

27 Gilligan P. Respiratory cultures from cystic fibrosis patients. In: Isenberg HD, ed. Clinical Microbiology Procedures Handbook. 2nd Edn. Washington, ASM Press, 2004; pp. 3.11.3.1-3.11.3.9.

28 Spencker FB, Haupt S, Claros MC, et al. Epidemiologic characterization of Pseudomonas aeruginosa in patients with cystic fibrosis. Clin Microbiol Infect 2000; 6: 600-607.

29 Baldwin A, Mahenthiralingam E, Drevinek P, et al. Elucidating global epidemiology of Burkholderia multivorans in cases of cystic fibrosis by multilocus sequence typing. J Clin Microbiol 2008; 46: 290-295.

30 Høiby N, Pedersen SS. Estimated risk of cross-infection with Pseudomonas aeruginosa in Danish cystic fibrosis patients. Acta Paediatr Scand 1989; 78: 395-404. 